\title{
Hot-electron dynamics at noble metal surfaces
}

\author{
C. López-Bastidas, ${ }^{1}$ J.A. Maytorena, ${ }^{1,2}$ and A. Liebsch ${ }^{1}$ \\ ${ }^{1}$ Institut für Festkörperforschung, Forschungszentrum Jülich, 52425 Jülich, Germany \\ ${ }^{2}$ Centro de Ciencias Físicas, Universidad Nacional Autónoma de México, Apartado Postal 48-3, 62251 Cuernavaca, Morelos, México
}

(Received 23 August 2001; published 28 December 2001)

\begin{abstract}
The dynamics of excited electronic states at Ag surfaces is studied by evaluating the quasiparticle selfenergy within the $G W$ approximation. The screened Coulomb interaction $W$ is shown to be sensitive to the spatial variation of $s-d$ screening near the surface. In the region of $s$-electron spill-out electronic damping is stronger than in the bulk due to the reduced $s-d$ polarization, giving rise to shorter surface-state lifetimes. The lifetime of Ag image states is expected to be strongly reduced due to decay into surface plasmons.
\end{abstract}

DOI: 10.1103/PhysRevB.65.035417

PACS number(s): 71.15.Mb, 73.50.Gr, 78.40.Kc

\section{INTRODUCTION}

The lifetime of quasiparticles is a quantity of central importance in solid-state physics. At surfaces, the decay of excited electron states plays a crucial role in processes such as atom-surface charge and energy transfer, hot-electroninduced molecular processes like desorption and dissociation of adsorbates, and other chemical reactions. ${ }^{1-4}$ The lifetime also determines the phase relaxation length, i.e., the distance a quasiparticle can travel without losing its phase memory. Using femto-second lasers and scanning tunneling microscopy it is now possible to study the real-time decay of excited states ${ }^{5,6}$ and to separate electronic processes from broadening due to phonons and defects.

An area that has received particular attention is the lifetime of image and surface states. ${ }^{7,8}$ In the case of noble metals, an interesting issue concerns the role of the screening interaction between extended $s p$ and localized $d$ electrons. Several recent experiments indicate that the spatial characteristics of the $s-d$ polarization in the surface region influence the dynamics of excited electrons, for example, the surface-state lifetime observed in scanning tunneling spectroscopy on $\mathrm{Ag},{ }^{9}$ the phase relaxation length observed in quantum-mechanical interference patterns near $\mathrm{Ag}$ step edges, ${ }^{10}$ and the size dependence of electronic interactions ${ }^{11}$ and of the frequency and width of the Mie plasmon ${ }^{12}$ in $\mathrm{Ag}$ nanoparticles.

The key quantity characterizing lifetime phenomena is the nonlocal complex self-energy which yields the relaxation shift and broadening of an excited state due to its interaction with the surrounding system. In view of the difficulty of performing self-energy calculations, few studies for realistic bulk metals have been performed so far. ${ }^{13-16}$ For metal surfaces, the jellium model ${ }^{17}$ and a one-dimensional potential ${ }^{18}$ focusing on the band structure perpendicular to the surface were used. The latter model provides valuable insight into various contributions to the lifetime of surface and image states on several systems. ${ }^{6,14,19}$ The effect of $s-d$ screening in noble metals, however, is included only approximately in this scheme. In particular, the spatial variation of $s-d$ polarization due to the different amounts of spill-out of $s$ - and $d$-electron densities into the vacuum is missing.

In this work we investigate the effect of $s-d$ screening on the damping rate of hot electrons at $\mathrm{Ag}$ surfaces by evaluat- ing the electron self-energy within the $G W$ approximation. ${ }^{20,13}$ Since a fully three-dimensional (3D) treatment of surface screening dynamics for metals involving $d$ electrons is computationally not yet feasible we use a simplified model to describe qualitatively the effect of $s-d$ polarization on the decay of excited electrons at the surface. We make use of the fact that the $s p$ bands exhibit nearly-free-electron character up to the onset of transitions involving $d$ bands $(\sim 4 \mathrm{eV})$. For energies below this onset, interband transitions contribute only as virtual excitations. The additional screening due to such transitions gives rise to a mutual polarization between the fluctuating $s$ - and $d$-electron densities. This suggests using a two-component $s-d$-electron model in which the $s$ electrons are treated as a semi-infinite jellium and the occupied $d$ bands are accounted for by the presence of a polarizable medium which extends up to a certain distance from the electron-gas edge. This model had proven quite useful to explain the origin of the anomalous dispersion of collective excitations on Ag surfaces ${ }^{21,22}$ and in small $\mathrm{Ag}$ particles. ${ }^{23,24}$

As shown below, the absence of $s-d$ screening in the spillout region of $s$-electron density leads to stronger effective Coulomb interactions than in the interior of the metal and to shorter electronic lifetimes, including those of surface states. Moreover, the lifetime depends strongly on frequency since above a certain threshold decay into surface collective modes is feasible. The opening of collective decay channels is found to be particularly important for the lifetime of $\mathrm{Ag}$ image states.

The paper is organized as follows. In Sec. II we describe the model and derive an expression for the dynamically screened Coulomb interaction. In Sec. III we present the results and in Sec. IV we discuss their relevance in relation to other theoretical and experimental works. A summary is given in Sec. V. Atomic units $(m=|e|=\hbar=1)$ are used throughout unless noted otherwise.

\section{THEORY}

The two-component model used in the present work is illustrated schematically in Fig. 1. The $s$ electrons are treated via a semi-infinite jellium while the presence of the occupied $d$ bands is taken into account via a polarizable medium which accounts for the polarization between $s$ and $d$ elec- 


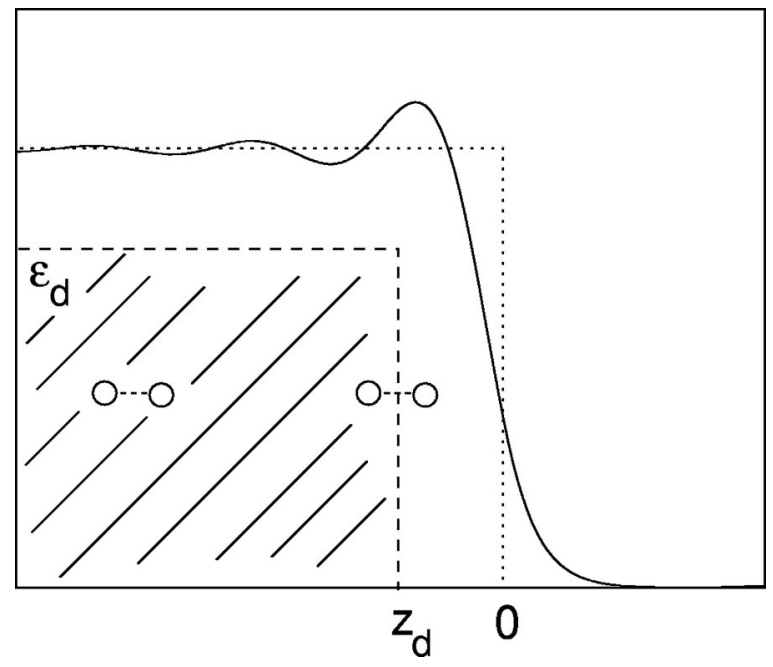

FIG. 1. Two-component model for reduced $s-d$ screening at noble-metal surface. Solid curve: ground-state $s$-electron density; dotted curve: positive background; dashed curve: polarizable $d$-electron medium.

trons. The dynamical response of the jellium is described using the nonlocal density response function derived within the local-density approximation (LDA). The polarizable medium is characterized by a local dielectric function $\epsilon_{d}(\omega)$ related to the total bulk dielectric-response function of the metal via $\epsilon(\omega)=\epsilon_{s}(\omega)+\epsilon_{d}(\omega)-1$, where $\epsilon_{s}(\omega)$ corresponds to the long-wavelength limit of the $s$-electron dielectric-response function. ${ }^{25}$

An important feature incorporated in this model is the fact that the vacuum spill-out of charge density stems primarily from the $s$ electrons rather than the more tightly bound $d$ electrons. Thus, the background of positive charge neutralizing the $s$-electron density is located in the half-space $z \leqslant 0$ while the polarizable medium extends only up to $z \leqslant z_{d}$. A $z$-dependent local dielectric function is defined as $\epsilon_{d}(z, \omega)$ $=\epsilon_{d}(\omega)$ for $z \leqslant z_{d}$ and $\epsilon_{d}(z, \omega)=1$ for $z>z_{d}$. As indicated in Fig. 1, $s$ electrons in the interior are subject to isotropic $d$-electron screening, while near the surface this screening is only partially present.

In this work, the $G W$ approximation is used to calculate the self-energy $\Sigma$ of a quasiparticle. Because of the translational invariance parallel to the surface it is convenient to perform a two-dimensional Fourier transform characterized by $\mathbf{q}_{\|}$. To derive an expression for the dynamically screened Coulomb interaction $W\left(z, z^{\prime}, q, \omega\right)\left(q=\left|\mathbf{q}_{\|}\right|\right)$, it is useful to relate a ficticious perturbing charge density $n_{\text {ext }}(z, q, \omega)$ to the total dynamic potential $\phi(z, q, \omega)$ via

$$
\phi(z, q, \omega)=\int d z^{\prime} W\left(z, z^{\prime}, q, \omega\right) n_{e x t}\left(z^{\prime}, q, \omega\right)
$$

We start by obtaining a general expression for $W$ within the random-phase approximation (RPA) and subsequently specify for the two-component $s$ - $d$-electron system. For clarity the $z, q$, and $\omega$ dependencies of quantities, as well as the integrals in response equations, are frequently omitted.
The perturbing charge generates the potential $\phi_{\text {ext }}$ $=K n_{\text {ext }}$, where $K\left(z, z^{\prime}, q\right)=(2 \pi / q) e^{-q\left|z-z^{\prime}\right|}$ is the bare Coulomb kernel. Within a self-consistent-field approach the total dynamic potential $\phi$ differs from the perturbing potential $\phi_{\text {ext }}$ because of screening processes. The total potential is the sum of the perturbing and induced potentials: $\phi$ $=\phi_{\text {ext }}+\phi_{\text {ind }}$. The induced charge density $n(z, q, \omega)$ is related to $\phi_{\text {ext }}$ via

$$
n(z, q, \omega)=\int d z^{\prime} \chi\left(z, z^{\prime}, q, \omega\right) \phi_{e x t}\left(z^{\prime}, q, \omega\right),
$$

where the susceptibility $\chi$ is the full many-body density response function of the $s-d$-electron system. Within the RPA the induced potential is given by $\phi_{i n d}=K n$ and the total dynamic potential can be written as

$$
\phi=K\left(n_{\text {ext }}+\chi \phi_{\text {ext }}\right)=(K+K \chi K) n_{\text {ext }} .
$$

Equations (1) and (3) imply the integral equation

$$
W=K+K \chi K .
$$

In the RPA the induced density equals that of a system of noninteracting electrons with susceptibility $\chi^{0}$ responding to the total dynamical potential $\phi$ :

$$
n(z, q, \omega)=\int d z^{\prime} \chi^{0}\left(z, z^{\prime}, q, \omega\right) \phi\left(z^{\prime}, q, \omega\right),
$$

which is equivalent to stating $\chi=\chi^{0}+\chi^{0} K \chi$. Therefore the screened Coulomb interaction is given by

$$
W=\left[1-K \chi^{0}\right]^{-1} K
$$

Within the two-component model, expression (6) can be reformulated in the following way. First, we note that the bare susceptibility can be separated into $s$ and $d$ contributions: $\chi^{0}=\chi_{s}^{0}+\chi_{d}^{0}$. This can be done since the noninteracting susceptibility involves a sum over occupied states whose nature ( $s$ or $d$ ) can be identified as long as there is only weak $s-d$ hybridization. It follows that the induced density can also be separated into $n=n_{s}+n_{d}$, where the contributions are given by

$$
n_{s, d}(z, q, \omega)=\int d z^{\prime} \chi_{s, d}^{0}\left(z, z^{\prime}, q, \omega\right) \phi\left(z^{\prime}, q, \omega\right) .
$$

The total dynamic potential obeys the Poisson equation $\phi^{\prime \prime}$ $-q^{2} \phi=-4 \pi n_{t o t}$, where $n_{t o t}=n_{e x t}+n_{s}+n_{d}$; the primes on $\phi$ denote derivative with respect to $z$.

In the following derivation the explicit dependence on $n_{d}$ is eliminated and an expression for the total induced density is found in terms of $n_{s}$ and the dielectric function of the semi-infinite polarizable medium. The objective is to obtain an expression for $W$ which involves only $\chi_{s}^{0}$ and $\epsilon_{d}(z, \omega)$.

With the introduction of the perturbing charge the $d$-electron medium acquires a polarization given by $4 \pi \mathbf{P}_{d}(z)=\left[\epsilon_{d}(z, \omega)-1\right] \nabla \phi(z)$, where the gradient operator is the vector $\nabla=\left(i \mathbf{q}_{\|}, \partial_{z}\right)$. The induced $d$-electron density is determined from $n_{d}=\nabla \cdot \mathbf{P}_{d}$. Using the Poisson equation, $n_{d}$ can be written as 


$$
n_{d}(z)=a \delta\left(z_{d}-z\right)-\frac{\epsilon_{d}(z, \omega)-1}{\epsilon_{d}(z, \omega)}\left[n_{s}(z)+n_{\text {ext }}(z)\right],
$$

where $a \equiv-P_{d}\left(z_{d}\right)$. The total density is then

$$
n_{t o t}(z)=\frac{n_{s}(z)+n_{e x t}(z)}{\epsilon_{d}(z, \omega)}+a \delta\left(z_{d}-z\right) .
$$

The first term gives the external and induced $s$-electron contributions screened due to the presence of $d$ electrons whereas the last term accounts for the $d$-screening charge at the boundary of the polarizable medium.

The potential $\phi=K n_{\text {tot }}$ is given by

$$
\begin{aligned}
\phi(z)= & \frac{2 \pi}{q} \int d z^{\prime} \frac{e^{-q\left|z-z^{\prime}\right|}}{\epsilon_{d}\left(z^{\prime}, \omega\right)}\left[n_{s}\left(z^{\prime}\right)+n_{e x t}\left(z^{\prime}\right)\right] \\
& +\frac{2 \pi}{q} a e^{-q\left|z-z_{d}\right|} .
\end{aligned}
$$

The coefficient $a$ can be determined from the continuity condition across the $z=z_{d}$ plane

$$
\epsilon_{d}(\omega) \phi^{\prime}\left(z_{d}^{-}\right)=\phi^{\prime}\left(z_{d}^{+}\right),
$$

which, restoring all arguments, yields

$$
\begin{aligned}
a(q, \omega)= & \sigma_{d}(\omega) \int d z^{\prime} \frac{e^{-q\left|z_{d}-z^{\prime}\right|}}{\epsilon_{d}\left(z^{\prime}, \omega\right)} \operatorname{sgn}\left(z_{d}-z^{\prime}\right)\left[n_{s}\left(z^{\prime}, q, \omega\right)\right. \\
& \left.+n_{\text {ext }}\left(z^{\prime}, q, \omega\right)\right],
\end{aligned}
$$

where $\sigma_{d}(\omega)=\left[\epsilon_{d}(\omega)-1\right] /\left[\epsilon_{d}(\omega)+1\right]$.

We now introduce a $d$-screened Coulomb kernel via

$$
\begin{aligned}
K^{\prime}\left(z, z^{\prime}, q, \omega\right)= & \frac{2 \pi}{q} \frac{1}{\epsilon_{d}\left(z^{\prime}, \omega\right)}\left[e^{-q\left|z-z^{\prime}\right|}+\operatorname{sgn}\left(z_{d}-z^{\prime}\right)\right. \\
& \left.\times \sigma_{d}(\omega) e^{-q\left|z-z_{d}\right|} e^{-q\left|z^{\prime}-z_{d}\right|}\right]
\end{aligned}
$$

such that the substitution of Eq. (12) in (10) gives

$$
\begin{aligned}
\phi(z, q, \omega)= & \int d z^{\prime} K^{\prime}\left(z, z^{\prime}, q, \omega\right)\left[n_{\text {ext }}\left(z^{\prime}, q, \omega\right)\right. \\
& \left.+n_{s}\left(z^{\prime}, q, \omega\right)\right] .
\end{aligned}
$$

The first term in Eq. (13) is the bare Coulomb interaction $K$ screened by the polarization charges induced within the polarizable background. The second term stems from polarization charges at the boundary of the medium.

Using Eqs. (7), (13), and (14) the induced s-electron density takes the form

$$
n_{s}=\left[1-\chi_{s}^{0} K^{\prime}\right]^{-1} \chi_{s}^{0} K^{\prime} n_{e x t} \equiv \chi_{S}^{\prime} K^{\prime} n_{\text {ext }}
$$

where $\chi_{s}^{\prime}=\left(1-\chi_{s}^{0} K^{\prime}\right)^{-1} \chi_{s}^{0}$ defines the total screened susceptibility. This expression accounts for the renormalization of the bare $s$ electron density response $\chi_{s}^{0}$ due to both $s$ - and $d$-electron screening. The dynamic potential can finally be cast in the form

$$
\phi=\left[K^{\prime}+K^{\prime} \chi_{s}^{\prime} K^{\prime}\right] n_{\text {ext }},
$$

from which, according to Eq. (1), the dynamic screened interaction $W$ can be identified. Thus, within the present model $W$ is given by

$$
W=K^{\prime}+K^{\prime} \chi_{s}^{\prime} K^{\prime} .
$$

Coupling to surface plasmons is included in this formalism and is associated with the poles of $\chi_{s}^{\prime}$.

We note here that $\operatorname{Im} W$ may be viewed as the absorptive part of the screened complex potential induced by a charge sheet located at $z^{\prime}$. Accordingly, the rate of generating electronic surface excitations by an externally reflected electron, which can be obtained from the so-called surface loss function $\operatorname{Im} g(q, \omega),{ }^{22}$ is given by the behavior of $\operatorname{Im} W\left(z, z^{\prime}\right)$ far from the surface. In the limit $z, z^{\prime} \gg 0$ we find $\operatorname{Im} W\left(z, z^{\prime}, q, \omega\right) \rightarrow-(2 \pi / q) e^{-q\left(z+z^{\prime}\right)} \operatorname{Im} g(q, \omega)$.

In the $G W$ approximation the self-energy $\Sigma$ is obtained using only the first-order term in a series expansion in terms of $W .^{13,20}$ The self-energy of an electron in an excited state $\psi_{i}(\mathbf{r})=\psi_{i}(z) e^{i \mathbf{k}_{\|} \cdot \mathbf{r}_{\|}}$with energy $E_{i}=\varepsilon_{i}+\frac{1}{2} \mathbf{k}_{\|}^{2}$ and parallel momentum $\mathbf{k}_{\|}$is given by ${ }^{19}$

$$
\begin{aligned}
\Sigma\left(z, z^{\prime}, k_{\|}, E_{i}\right)= & \int \frac{d E}{2 \pi} \int \frac{d \mathbf{q}_{\|}}{(2 \pi)^{2}} i G\left(z, z^{\prime}, \mathbf{k}_{\|}-\mathbf{q}_{\|}, E_{i}-E\right) \\
& \times W\left(z, z^{\prime}, q_{\|}, E\right) .
\end{aligned}
$$

$\varepsilon_{i}$ is the energy associated with motion normal to the surface. In accordance with the RPA for the calculation of the susceptibilities, the interacting Green function $G$ is replaced by the noninteracting $G_{0}$ to obtain

$$
\operatorname{Im} \Sigma\left(z, z^{\prime}, k_{\|}, \omega\right)=\sum_{\varepsilon_{f}, \mathbf{q}_{\|}} \psi_{f}^{*}(z) \operatorname{Im} W\left(z, z^{\prime}, q_{\|}, \omega^{\prime}\right) \psi_{f}\left(z^{\prime}\right)
$$

where $\omega=E_{i}-E_{F}$ and $\psi_{f}(z) e^{i\left(\mathbf{k}_{\|}+\mathbf{q}_{\|}\right) \cdot \mathbf{r}_{\|}}$are single-particle $s$-electron wave functions of the available final states with energy $E_{f}=\varepsilon_{f}+\frac{1}{2}\left(\mathbf{k}_{\|}+\mathbf{q}_{\|}\right)^{2}$ to which the electron may scatter. From energy and momentum conservation it follows that the summation over the final states is subject to the condition $0 \leqslant \omega^{\prime} \leqslant \omega$, where $\omega^{\prime}=E_{i}-E_{f}$ is the energy transfer.

The damping rate (or linewidth) of the excited state $\psi_{i}$ is given by

$$
\tau^{-1}(\omega)=-2 \int d z \int d z^{\prime} \psi_{i}^{*}(z) \operatorname{Im} \Sigma\left(z, z^{\prime}, k_{\|}, \omega\right) \psi_{i}\left(z^{\prime}\right) .
$$

\section{RESULTS}

In this section we discuss results for Ag. The dynamical $s$-electron response is derived for a semi-infinite jellium of bulk density $n_{s}=3 / 4 \pi r_{s}^{3}$ with $r_{s}=2.97 a_{0} \quad\left(a_{0}=0.529 \AA\right.$ is the Bohr radius). The ground-state properties are obtained using the LDA. An average pseudopotential of $V_{0}=$ $-2.9 \mathrm{eV}$ representing the effect of the $d$ bands is included in order to reproduce the correct work function of about 4.5 


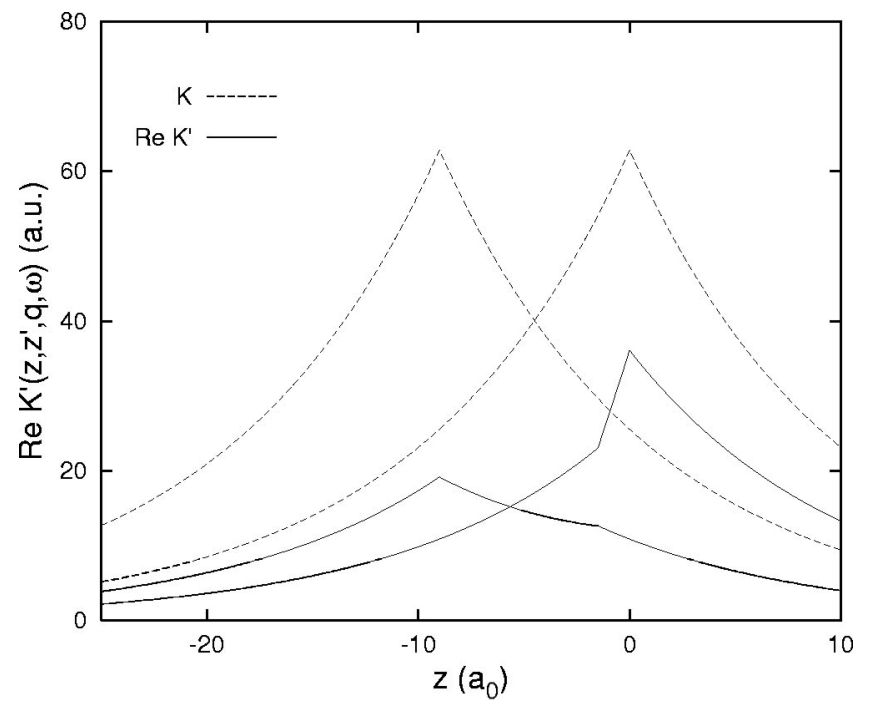

FIG. 2. Solid curve: Coulomb interaction kernel screened by polarizable medium $K^{\prime}\left(z, z^{\prime}, q, \omega\right)$ for $\omega=1 \mathrm{eV}$ and dashed curve: unscreened interaction $K\left(z, z^{\prime}, q\right)$; both are at $q=0.1 a_{0}^{-1}$ and for $z_{d}=-1.5 a_{0}$. To illustrate the interaction near the surface and in the interior, results are shown for $z^{\prime}=0$ (left curves) and $-9 a_{0}$ (right curves).

eV. The final states appearing in Eq. (19) as $\psi_{f}(z)$ are eigenfunctions of a one-dimensional Kohn-Sham Hamiltonian with total potential $V(z)=V_{e f f}(z)+V_{0} \theta(-z)$. The first term is the one-electron potential $V_{\text {eff }}(z)=V_{+}(z)+V_{\text {Hartree }}(z)$ $+V_{x c}(z)$, where $V_{+}$is due to the semi-infinite background of positive charge and $V_{\text {Hartree }}$ is the electrostatic potential associated to the electron density. The contribution $V_{x c}$ is derived from the Wigner formula for the exchangecorrelation energy.

The local dielectric function $\epsilon_{d}(\omega)$ is taken from bulk optical data. ${ }^{26}$ The boundary $z_{d}=-1.5 a_{0}$ used in this work was previously found to best reproduce the surface-plasmon dispersion relation for Ag surfaces. ${ }^{21}$ Results for $z_{d}=0$ are also shown for comparison.

In Fig. 2, the $d$-screened Coulomb interaction $K^{\prime}\left(z, z^{\prime}, q, \omega\right)$ defined in Eq. (13) is compared with the bare Coulomb kernel $K\left(z, z^{\prime}, q\right)$. Through $\epsilon_{d}(\omega, z)$ the former depends on frequency. Below the onset of interband transitions $(\approx 3.9 \mathrm{eV}) \epsilon_{d}(\omega)$ is real and therefore $\operatorname{Im} K^{\prime} \approx 0$. In general $K^{\prime}$ is reduced compared to $K$. In a spatially uniform system, i.e., deep in the bulk, the second term in Eq. (13) is small so that $K^{\prime} \rightarrow K / \epsilon_{d}(\omega)$. Due to the semi-infinite nature of the $d$-electron medium $K^{\prime}$ exhibits a more complicated dependence on both $\epsilon_{d}(\omega)$ and $z_{d}$ for $z$ or $z^{\prime}$ near the surface. Since for $\operatorname{Ag} \sigma_{d} \approx 1 / 2 \cdots 2 / 3$, the polarization charges at the boundary of the medium cause a significant modification of the magnitude and spatial variation of the Coulomb interaction in the surface region. For $z, z^{\prime} \gg 0, K^{\prime} \rightarrow K$. The exponential decay for $z \neq z^{\prime}$ both in the bulk and at the surface is determined by $q$. When $z=z_{d}$ the derivative of $K^{\prime}$ has a discontinuity since the polarizable background ends at $z_{d}$; when $z^{\prime}>z_{d}$ the derivative increases while the opposite is true for $z^{\prime}<z_{d}$ due to the sign function in Eq. (13).

Figure 3 shows the real part of the $s$ - $d$-screened interac-

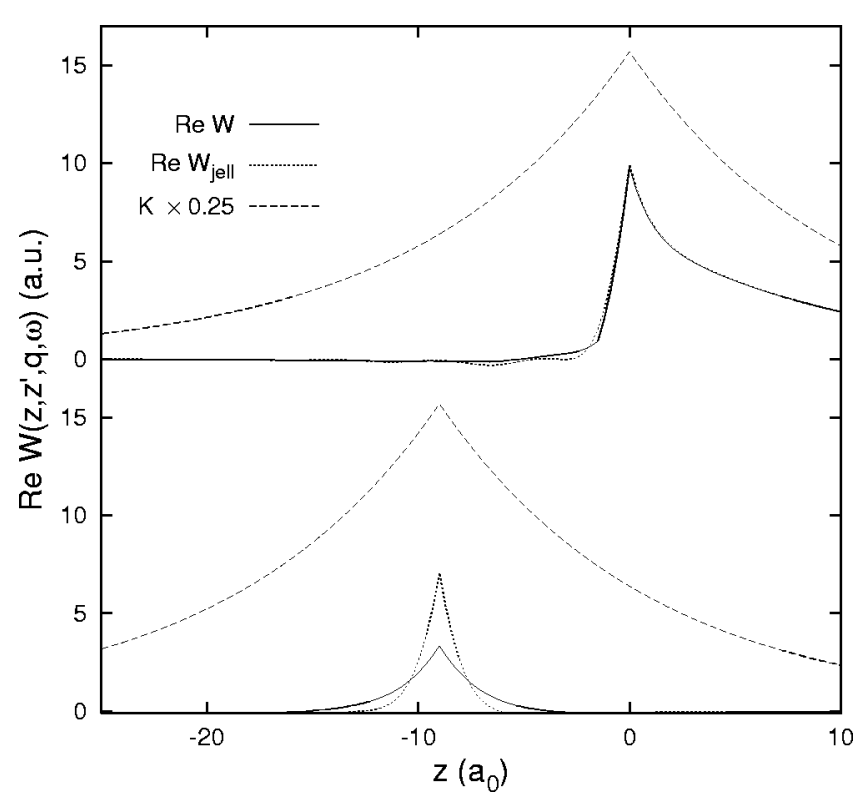

FIG. 3. Real part of dynamically screened Coulomb interaction $W$ for $\omega=1 \mathrm{eV}, q=0.1 a_{0}^{-1}$, and $z_{d}=-1.5 a_{0}$. Upper panel: $z^{\prime}$ $=0$ ("surface screening"); lower panel: $z^{\prime}=-9 a_{0}$ ("bulk screening"). Solid curves: two-component $s$ - $d$-electron model; dotted curves: bare jellium model; dashed curves: unscreened Coulomb kernel $K$.

tion $W$, calculated from Eq. (17), and the bare interaction $K$. For comparison we also show $W_{\text {jell }}$ which is the screened interaction of a jellium system with the same $s$-electron density as Ag. In contrast to $K$, the screened interaction kernels $W$ and $W_{\text {jell }}$ depend nonlocally on the density of the electron gas. The asymmetric shape close to the surface (see top panel of Fig. 3) is due to a reduction of screening in the region of $s$-electron spill-out. The screened interaction $W$ approximates $W_{\text {jell }}$ in the surface region given the reduced $d$-electron density whereas in the bulk, $s-d$ screening is isotropic. Well inside the metal $\left(z, z^{\prime} \ll 0\right), W_{\text {jell }}\left(z, z^{\prime}, q, \omega\right)$ agrees with the equivalent RPA expression for a homogeneous electron gas. In this case, the 3D Fourier transform of the bulk screened Coulomb interaction is given by $W_{j e l l}^{b}(p, \omega)$ $=v(p) / \epsilon_{L}(p, \omega)$, where $v(p)=4 \pi / p^{2}$ is the bare Coulomb interaction, $\epsilon_{L}$ the Lindhard dielectric function, and $p$ is the magnitude of a three-dimensional wave vector. In the presence of a homogeneous $d$-electron medium, the effective interaction in the bulk becomes $W^{b}(p, \omega)=v(p) /\left[\epsilon_{L}(p, \omega)\right.$ $\left.+\epsilon_{d}(\omega)-1\right]$. Performing a one-dimensional Fourier transform then yields $W^{b}\left(z, z^{\prime}, q, \omega\right)$ which coincides with the screened interaction $W\left(z, z^{\prime}, q, \omega\right)$ [Eq. (17)] in the limit $z, z^{\prime} \ll 0$. As evident in Fig. 3 , in the bulk, $W$ decays more slowly than $W_{\text {jell }}$ because of the larger effective screening length.

The results shown in Fig. 3 are typical for low frequencies. At higher frequencies collective excitations modify the picture, making $W$ about an order of magnitude larger. The Ag surface plasma frequency at $q=0$ is $\omega_{s}=\omega_{p} / \sqrt{\epsilon_{d}+1}$ $\approx 3.7 \mathrm{eV}$, where $\omega_{p}=9.2 \mathrm{eV}$ is the $s$-electron bulk plasma frequency. For the jellium the corresponding value is $\omega_{s}$ $=6.5 \mathrm{eV}$. 


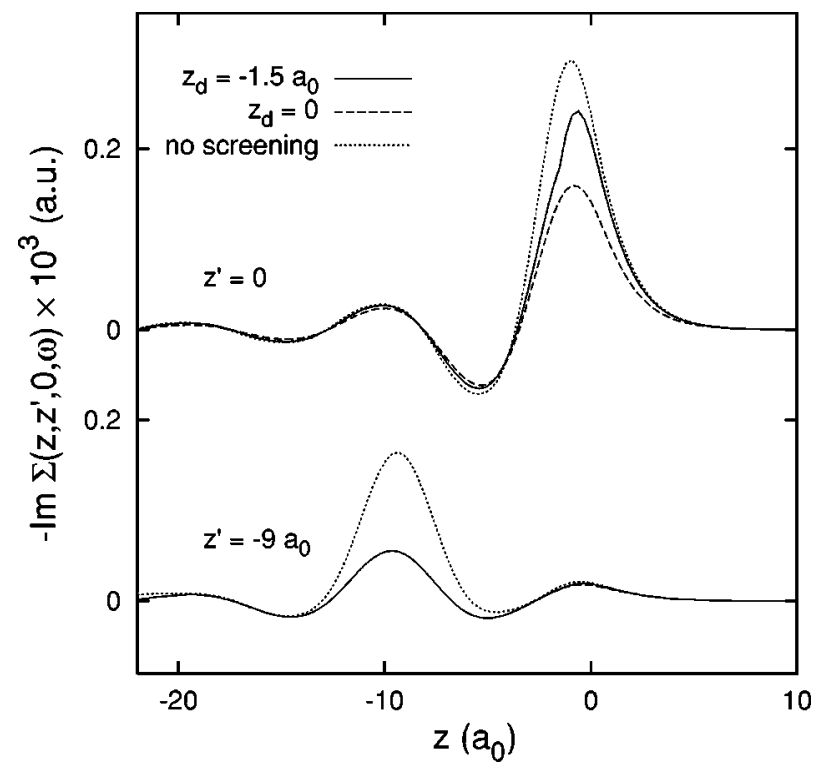

FIG. 4. Imaginary part of self-energy $\Sigma\left(z, z^{\prime}, k_{\|}, \omega\right)$ for Ag surface $\left(k_{\|}=0, \omega=1 \mathrm{eV}\right)$. Upper panel: $z^{\prime}=0$ ("surface screening"); lower panel: $z^{\prime}=-9 a_{0}$ ("bulk screening").

Below the onset of interband transitions the imaginary part of $W$ is determined by the imaginary part of $\chi_{s}^{\prime}$. At low frequencies $\operatorname{Im} W$ is mainly due to electron-hole pair excitation. It exhibits a similar surface-to-bulk relation as the real part. Above the surface plasma energy $\operatorname{Im} W$ becomes an order of magnitude larger and spatially extended. For $z, z^{\prime}$ $\gg 0$ the position of the resonance within the $q-\omega$ plane coincides with the peak of the surface loss function $\operatorname{Im} g(q, \omega) .^{21,22}$

To obtain the imaginary part of the self-energy of an excited state of energy $E_{i}$, a sum over the available phase space of final unoccupied states must be done. In this work we have considered initial states with zero parallel momentum; the variation with $k_{\|}$has been shown to be weak. ${ }^{17,19}$ The sums in Eq. (19) can be transformed into a threedimensional integration. The integration was done in spherical coordinates $(k, \theta, \varphi)$, where the one corresponding to the azimuthal angle $\varphi$ can be done analytically. The symmetry of the integrand permits the polar angle $\theta$ to be constrained from 0 to $\pi / 2$ and the wave-vector magnitude $k$ from $k_{F}$ to $\sqrt{2 E_{i}}$. The integrand is maximum for $k=k_{F}$ which corresponds to $\omega^{\prime}=E_{i}-E_{F}$, and zero for $k=\sqrt{2 E_{i}}$ which corresponds to $\omega^{\prime}=0$. The integration involves the product $q W\left(z, z^{\prime}, q, \omega^{\prime}\right)$ for values of $q=k \sin \theta$ from 0 to $\sqrt{2 E_{i}}$. The behavior at small $q$ was extrapolated from $q=0.02 a_{0}^{-1}$ which is attainable with our numerical implementation. The contribution to the self-energy due to the surface-plasmon resonance of $W$ can be very large even though the phase space involved is a small fraction of the total.

Figure 4 shows the imaginary part of the self-energy for $\mathrm{Ag}$ at $1-\mathrm{eV}$ excitation energy. According to Eq. (20), the lifetime of the state $\psi_{i}$ is inversely related to the overall magnitude of $-\operatorname{Im} \Sigma$. As a result of $s-d$ screening, the $\mathrm{Ag}$ self-energy is seen to be reduced relative to the corresponding self-energy in the absence of $d$ electrons. This reduction is strongest in the interior $\left(z^{\prime} \ll 0\right)$ since an excited $s$ electron perceives a nearly isotropic polarizable $d$-electron density. ${ }^{27}$ An $s$ electron forming part of the density spill-out near the surface, however, is less subject to $s-d$ screening because of the confinement of the $d$-electron density. This effect implies shorter lifetimes of low-lying surface states than in a model based on uniform $d$-electron screening.

An attractive feature of our model is that we can tune the range of $s-d$ polarization by adjusting the boundary $z_{d}$ of the $d$-electron medium. According to the results shown in Fig. 4 for $z_{d}=0, s-d$ screening is important even for $z^{\prime}$ near the surface so that the self-energy differs quite strongly from the $s$-electron case. Conversely, for $z_{d}<0$ the region of reduced $s-d$ screening increases and the self-energy near the surface begins to resemble the unscreened $s$-electron self-energy. As stated above, previous work ${ }^{21}$ indicated that the Ag surfaceplasmon dispersion is well described by choosing $z_{d} \approx$ $-1.5 a_{0}$. This suggests that at low excitation frequencies the electronic damping at $\mathrm{Ag}$ surfaces is only weakly reduced due to $s$ - $d$ screening. Thus, surface damping is very much stronger than bulk damping.

At simple metal surfaces the lifetime is, of course, also shorter than in the bulk because of efficient creation of electron-hole pairs in the region of the potential barrier. For the example shown in Fig. 4 , in the absence of $s-d$ polarization the amplitude of the surface peak of $\operatorname{Im} \Sigma$ is $50 \%$ larger than the bulk peak. Including $s-d$ screening, however, the difference is a factor of 4 since for $z_{d}=-1.5 a_{0}$ the surface peak is only weakly reduced while the bulk peak is subject to full $s-d$ screening.

The results shown in Fig. 4 are typical of the $s-d$ screening effects at low frequencies far below the onset of interband transitions. In agreement with standard phase-space arguments, the imaginary part of the self-energy increases quadratically with $\omega$, just as in the absence of $d$ electrons. The quadratic coefficient, however, is much smaller and the overall size of the quadratic region is reduced (see below). The reason for the latter effect is the opening of new decay channels associated with surface collective excitations. As shown in Fig. 5, the coupling to these modes causes a striking enhancement of $\operatorname{Im} \Sigma$. Although the phase-space region with efficient coupling to surface plasmons is much smaller than the region of electron-hole pair creation, the strength of the plasmon peak at small $q$ is so large that it dominates $\operatorname{Im} \Sigma$ in this frequency range. In fact, for $z_{d}<0$ the surface self-energy $\left(z^{\prime}=0\right)$ is even larger than in the absence of $s-d$ screening, giving greatly reduced quasiparticle lifetimes.

As can also be seen in Fig. 5, the degree of nonlocality of the self-energy is significantly larger in the plasmon region than at low frequencies. In the latter case, spatial distributions of dynamical quantities such as the fluctuating density, the screened Coulomb interaction, and the self-energy generally are determined by a typical screening length which also governs the shape of the equilibrium density at the surface. Close to the Ag collective surface modes, however, this range increases significantly because of the larger penetration depth of the plasmon charge density and the concommitant less efficient screening. The dynamical Friedel oscilla- 


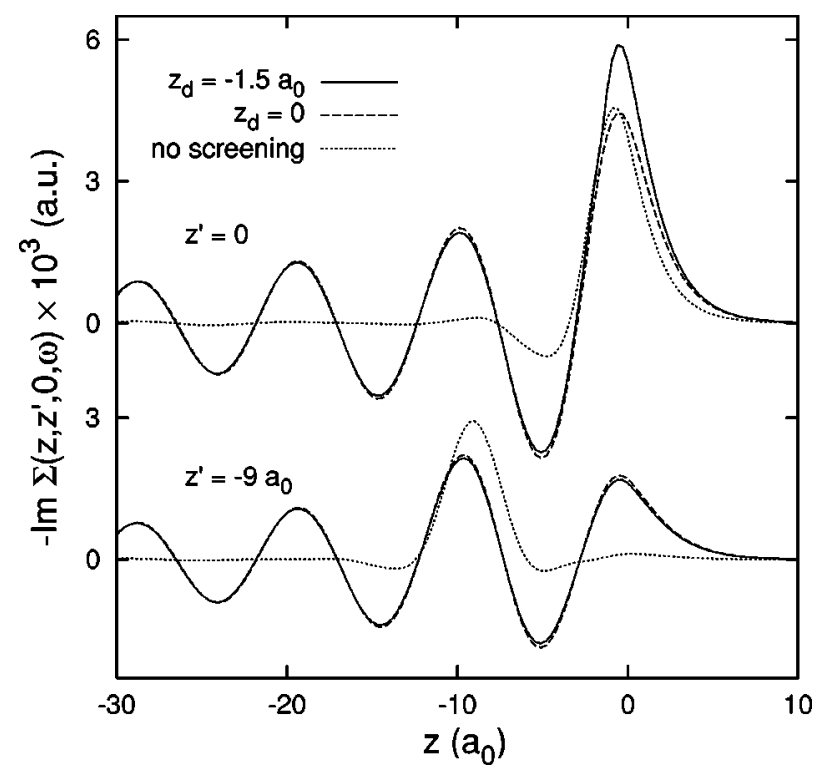

FIG. 5. Imaginary part of self-energy $\Sigma\left(z, z^{\prime}, k_{\|}, \omega\right)$ for Ag surface $\left(k_{\|}=0, \omega=4 \mathrm{eV}\right)$. Upper curves: $z^{\prime}=0$; lower panel: $z^{\prime}=$ $-9 a_{0}$.

tions then become very large and form an important part of the fluctuating charge density.

The surface-plasmon-induced enhancement of the $\mathrm{Ag}$ self-energy is illustrated in more detail in Fig. 6 which shows the frequency dependence of the maximum of $-\operatorname{Im} \Sigma$ for $z$ $=z^{\prime}=0$ and $-9 a_{0}$. At low excitation energies, the Ag selfenergy is smaller than for bare jellium because the effective Coulomb interaction $W$ is reduced due to $s-d$ screening. For $\omega>3.5 \mathrm{eV}$, however, the Ag self-energy is greatly enhanced due to excitation of surface plasmons. For bare jellium, analogous decay channels would appear above $\omega_{p} / \sqrt{2}$

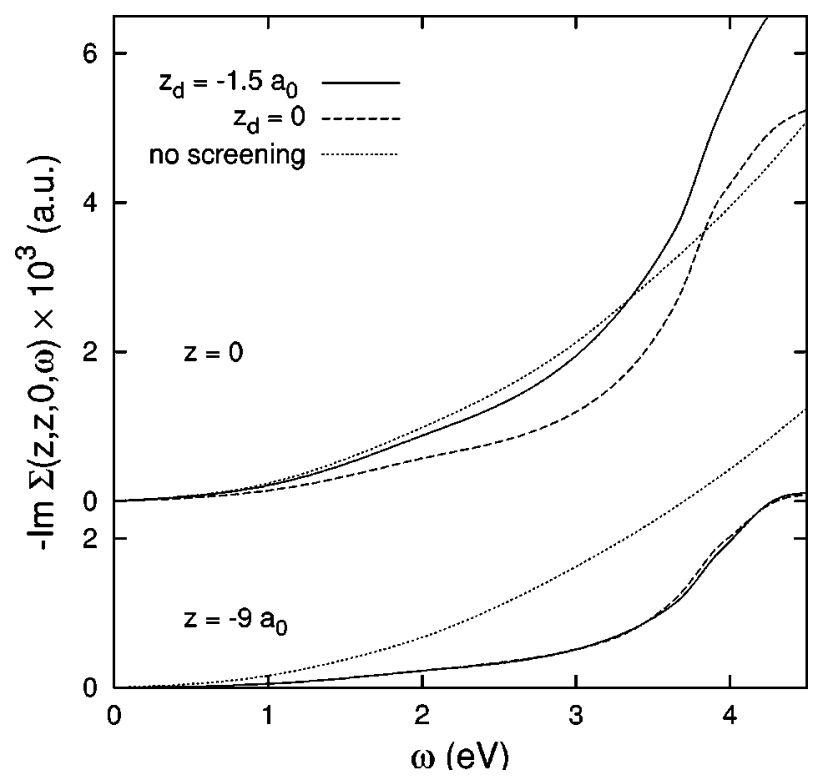

FIG. 6. Frequency dependence of imaginary part of self-energy $\Sigma(z, z, 0, \omega)$ for Ag surface. Upper curves: $z=0$; lower panel: $z=$ $-9 a_{0}$.

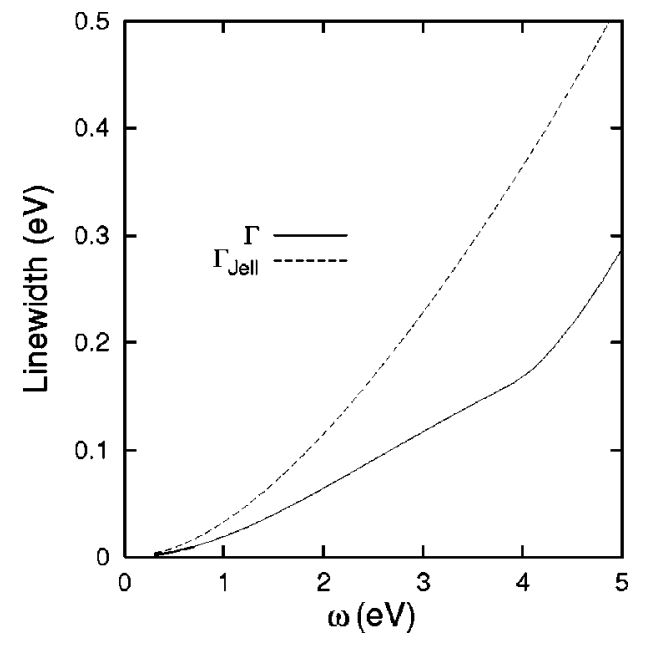

FIG. 7. Damping of excited electrons in bulk Ag as a function of energy above the Fermi level as calculated in the RPA. Solid curve: two-component $s$ - $d$ polarization model; dashed curve: ordinary jellium model.

$\approx 6.5 \mathrm{eV}$. Again, the details of the Ag self-energy near the surface depend sensitively on the spatial variation of the $s-d$ polarization. Thus, for $z_{d}=-1.5 a_{0}, s-d$ screening in the surface region is weaker than for $z_{d}=0$, giving a correspondingly larger self-energy.

\section{DISCUSSION}

The two-component $s$ - $d$ polarization model discussed in the present work reduces in the bulk limit, i.e., for $z, z^{\prime} \ll 0$, to the RPA treatment proposed first by Quinn ${ }^{32}$ for the evaluation of quasiparticle lifetimes in the presence of polarizable $d$ states. As discussed above, the screening of the Coulomb interaction via bound electrons decreases the imaginary part of the self-energy and enhances the quasiparticle lifetime. In Fig. 7, this effect is illustrated for bulk Ag. Plotted is the lifetime broadening with and without $d$-electron screening, $\Gamma(\omega)$ and $\Gamma_{\text {jell }}(\omega)$, respectively. Within $10 \%$ accuracy these quantities are related via $\Gamma(\omega) \approx \Gamma_{j e l l}(\omega) / \sqrt{\epsilon_{d}(\omega)}$. At low frequencies $\Gamma_{\text {jell }}(\omega)$ has the well-known quadratic behavior, where the coefficient depends only on the electron-density parameter $r_{s},{ }^{30,31}$ i.e., $\Gamma_{j e l l}(\omega)=\Gamma_{0}\left(r_{s}\right) \omega^{2} . \Gamma(\omega)$ also varies quadratically at low frequencies, but with a smaller coefficient. The change in the slope of $\Gamma(\omega)$ near $4 \mathrm{eV}$ is due to the onset of interband transition, i.e., due to the finite imaginary part of $\epsilon_{d}(\omega)$. One would expect a similar effect to be seen in experimental lifetime measurements and firstprinciples calculations.

Recent $G W$ calculations of the quasiparticle damping in bulk noble metals ${ }^{16}$ based on the LAPW electronic structure method yield results comparable to $\Gamma_{\text {jell }}(\omega)$ for $r_{s}=2.47 a_{0}$. This reduction is presumably associated with $s$ - $d$ electron screening below the interband onset. On the other hand, experimental measurements ${ }^{28,29}$ of the linewidth appear to agree with $\Gamma_{j e l l}(\omega)$ for $r_{s}=3 a_{0}$, as though $s$ - $d$ screening did not play a role. It should be noted, however, that the analysis of measured lifetimes is nontrivial due to impurity, 
phonon, and surface contributions. Moreover, depending on the type of spectroscopy used, generation of secondary electrons and transport away from the excitation region can influence the measured lifetimes.

The linewidth of surface states of noble metals was recently calculated ${ }^{6}$ using the $G W$ approach for a onedimensional potential model which simulates band-structure effects in the direction normal to the surface plane. Screening via $d$ electrons was treated phenomenologically via an overlap coefficient, which accounts for the penetration of the state's wave function into the bulk. ${ }^{7,33}$ For $\mathrm{Ag}(111)$ the calculated hole lifetime was found to be in good agreement with experimental results. Nevertheless, improvements in the treatment of the electronic and phonon contributions to the linewidth should lead to a better understanding of the relevant decay mechanisms. In particular, the model discussed in the present work provides a more refined manner of including the spatial variation of the $s-d$ polarization in the surface region. We emphasize that the results discussed in Sec. III for $\operatorname{Im} \Sigma$ are a consequence of the finite extent of the $d$-electron states. A spatially uniform $d$-electron medium corresponds instead to $z_{d} \gg 0$ and implies equally strong $s-d$ screening near the surface and in the bulk. The neglect of the spatial variation of $s$ - $d$ polarization therefore leads to an overestimate of electronic lifetimes near the surface. According to Fig. 4 states localized in the surface region are clearly much less affected by $s-d$ screening than states inside the metal. Also, a uniform $\epsilon_{d}(\omega)$ gives a too low surface plasma frequency $\omega_{p} / \sqrt{2 \epsilon_{d}}=2.7 \mathrm{eV}$ and therefore leads to an incorrect frequency dependence of the self-energy.

In considering image states at $\mathrm{Ag}$ surfaces whose energy above the Fermi level is nearly degenerate with the frequency of collective surface excitations it is expected that their lifetime should be shorter compared to results obtained in the absence of collective decay channels. In fact, an interesting crossover should be observable from the width of the early members of the Rydberg series to that of the higher image levels since their orbital overlap with the surfaceplasmon-induced self-energy decreases rapidly.

The results discussed above are relevant also for the size dependence of electron-electron interactions in noble-metal particles. With decreasing radius, the relative importance of the spill-out region of $s$-electron charge with reduced $s-d$ polarization increases, leading to a stronger effective Cou- lomb interaction and shorter hot-electron lifetimes. This has recently been observed by Voisin et al. ${ }^{11}$ The same mechanism also causes the blueshift and broadening of the Mie plasmon peak with decreasing radius. ${ }^{12,23,24,34}$

\section{CONCLUSIONS}

The approach discussed in the present work provides a qualitative picture of the effect of spatially varying $s-d$ screening on the quasiparticle dynamics at noble-metal surfaces. The main excitation mechanism included so far is the creation of electron-hole pairs and collective modes induced by the gradients of the time-varying fields in the surface region. To calculate the lifetime of specific surface and image states, it is necessary to account also for one-electron and matrix element effects arising from the band structure ignored in the jellium model. This could be achieved, for example, by treating the $s$ electrons within the one-dimensional potential model proposed by Chulkov et al. ${ }^{33}$ Such an extension would include the effect of the band structure perpendicular to the surface on the electronic transitions involved in the screened Coulomb interaction $W$. Equivalently, $s-d$ screening could also be incorporated in the slab approach ${ }^{17,19}$ by using the modified Coulomb kernel defined in Eq. (13). Work in these directions is currently under investigation and will be discussed in a future publication. ${ }^{35}$

In summary, the effect of $s-d$ screening on the lifetime of excited electrons at noble-metal surfaces is studied within the $G W$ approximation for a two-component $s-d$-electron model. The spatial variation of the mutual polarization between induced $s$ - and $d$-electron densities is shown to lead to an enhanced effective Coulomb interaction near the surface and to an accordingly larger imaginary self-energy. Thus, in the region of $s$-electron spill-out near the surface the quasiparticle damping is significantly larger than in the bulk because of weaker $s-d$ screening. A further enhancement is predicted at higher frequencies due to decay into surface plasmons. These results are expected to have important consequences for the lifetime of both low-lying surface states and image states near the vacuum threshold.

\section{ACKNOWLEDGMENTS}

This work was partially supported by CONACYT, México.
${ }^{1}$ J.W. Gadzuk, Chem. Phys. 251(1-3), 87 (2000).

${ }^{2}$ Laser Spectroscopy and Photochemistry on Metal Surfaces, edited by H.L. Dai and W. Ho (World Scientific, Singapore, 1995).

${ }^{3}$ See special issue of Chem. Phys. 251(1-3), 1 (2000).

${ }^{4}$ M. Wolf and G. Ertl, Science 288, 1352 (2000); E.W. Plummer, ibid. 277, 1447 (1997).

${ }^{5}$ H. Petek and S. Ogawa, Prog. Surf. Sci. 56, 239 (1997).

${ }^{6}$ J. Kliewer, R. Berndt, E.V. Chulkov, V.M. Silkin, P.M. Echenique, and S. Crampin, Science 288, 1399 (2000).

${ }^{7}$ Th. Fauster and W. Steinmann, in Photonic Probes of Surfaces, edited by P. Halevi (Elsevier, Amsterdam, 1995).
${ }^{8}$ R. Matzdorf, Surf. Sci. Rep. 30, 153 (1998); Chem. Phys. 251(13), 151 (2000).

${ }^{9}$ J. Li, W.D. Schneider, R. Berndt, O.R. Bryant, and S. Crampin, Phys. Rev. Lett. 81, 4464 (1998); R. Berndt, J. Li, W.D. Schneider, and S. Crampin, Appl. Phys. A: Mater. Sci. Process. 69, 503 (1999).

${ }^{10}$ L. Bürgi, O. Jeandupeux, H. Brune, and K. Kern, Phys. Rev. Lett. 82, 4516 (1999).

${ }^{11}$ C. Voisin, D. Cristofilos, N. Del Fatti, F. Vallée, B. Prével, E. Cottancin, J. Lermé, M. Pellarin, and M. Broyer, Phys. Rev. Lett. 85, 2200 (2000). 
${ }^{12}$ N. Nilius, N. Ernst, and H.-J. Freund, Phys. Rev. Lett. 84, 3994 (2000)

${ }^{13}$ F. Aryasetiawan and O. Gunnarsson, Rep. Prog. Phys. 61, 237 (1998).

${ }^{14}$ P.M. Echenique, J.N. Pitarke, E.V. Chulkov, and A. Rubio, Chem. Phys. 251, 1 (2000).

${ }^{15}$ I. Campillo, A. Rubio, J.M. Pitarke, A. Goldmann, and P.M. Echenique, Phys. Rev. Lett. 85, 3241 (2000); I. Campillo, V.M. Silkin, J.M. Pitarke, E.V. Chulkov, A. Rubio, and P. Echenique, Phys. Rev. B 61, 13484 (2000); I. Campillo, J.M. Pitarke, A. Rubio, E. Zarate, and P.M. Echenique, Phys. Rev. Lett. 83, 2230 (1999).

${ }^{16}$ R. Keyling, W.-D. Schöne, and W. Ekardt, Phys. Rev. B 61, 1670 (2000); W.-D. Schöne, R. Keyling, M. Bandic, and W. Ekardt, ibid. 60, 8616 (1999).

${ }^{17}$ J.J. Deisz and A. Eguiluz, Phys. Rev. B 55, 9195 (1997).

${ }^{18}$ E.V. Chulkov, V.M. Silkin, and P.M. Echenique, Surf. Sci. 437, 330 (1999).

${ }^{19}$ E.V. Chulkov, I. Sarria, V.M. Silkin, J.M. Pitarke, and P.M. Echenique, Phys. Rev. Lett. 80, 4947 (1998); I. Sarria, J. Osma, E.V. Chulkov, J.M. Pitarke, and P.M. Echenique, Phys. Rev. B 60, 11795 (1999); J. Osma, I. Sarria, E.V. Chulkov, J.M. Pitarke, and P.M. Echenique, ibid. 59, 10591 (1999).

${ }^{20}$ L. Hedin and S. Lundqvist, Solid State Phys. 23, 1 (1969).

${ }^{21}$ A. Liebsch, Phys. Rev. Lett. 71, 145 (1993); Phys. Rev. B 48, 11317 (1993).

${ }^{22}$ A. Liebsch, Electronic Excitations at Metal Surfaces (Plenum, New York, 1997), p. 92.
${ }^{23}$ J. Tiggesbäumker, L. Köller, K.H. Meiwes-Broer, and A. Liebsch, Phys. Rev. A 48, R1749 (1993).

${ }^{24}$ Ll. Serra and A. Rubio, Z. Phys. D: At., Mol. Clusters 40, 262 (1997).

${ }^{25}$ H. Ehrenreich and H.R. Philipp, Phys. Rev. 128, 1622 (1962).

${ }^{26}$ P.B. Johnson and R.W. Christy, Phys. Rev. B 6, 4370 (1972).

${ }^{27}$ Evidence for bulk screening effects on the lifetime was observed by S. Ogawa, H. Nagano, and H. Petek, Phys. Rev. B 55, 10869 (1997); See also Refs. 28,29,15 and 16.

${ }^{28}$ M. Wolf and M. Aeschlimann, Phys. Bl. 54, 145 (1998); M. Aeschlimann, M. Bauer, and S. Pawlik, Chem. Phys. 205, 127 (1996); E. Knoesel, A. Hotzel, T. Hertel, M. Wolf, and G. Ertl, Surf. Sci. 368, 76 (1996).

${ }^{29}$ J.J. Paggel, T. Miller, and T.-C. Chiang, Science 283, 1709 (1999).

${ }^{30}$ J.J. Quinn, Phys. Rev. 126, 1453 (1962); J.J. Quinn and R.F. Ferrell, ibid. 112, 812 (1958).

${ }^{31}$ D. Pines and P. Nozieres, The Theory of Quantum Liquids (Benjamin, New York, 1966).

${ }^{32}$ J.J. Quinn, Appl. Phys. Lett. 2, 167 (1963).

${ }^{33}$ E.V. Chulkov, V.M. Silkin, and P.M. Echenique, Surf. Sci. 391, L1217 (1997).

${ }^{34}$ Decay via surface collective excitations in small metal particles was recently studied by T.V. Shahbazyan and I.E. Perakis, Chem. Phys. 251, 37 (2000). However, the different amounts of $s$ - and $d$-electron spill-out were not included.

${ }^{35}$ A.García-Lekue, E.V. Chulkov, P. Echenique, and A. Liebsch (unpublished). 